\title{
Workspace and Kinematics Analysis of Redundant Space Manipulator
}

\author{
Jian-xia Zhang , Xiao-peng Wei \\ School of Mechanical Engineering, Dalian University of Technology, 116024 Dalian, China
}

\begin{abstract}
In order to meet the requirements of tasks in the complex space environment, we designed a kind of 7 degrees of freedom redundant space manipulator in this paper. Its 3D modelling is based on SolidWorks 2015. The joint coordinate system of redundant space manipulator is described with the Denavit-Hartenberg (D-H) method. And the kinematic model is established. The direct kinematic equations of redundant space manipulator are derived by the homogeneous coordinate transforming matrices. The workspace is simulated and analysed using the Monte Carlo method. The experimental results showed that the workspace of 7 degrees of freedom redundant space manipulator, which was designed in this paper, changed smoothly. What's more, it had not the abrupt phenomenon, and the independent part is bigger. Thus it verifies the rationality of our designing about the redundant space manipulator. It provides a good reference for the path planning and obstacle avoidance planning.
\end{abstract}

\section{Introduction}

With the development of the aerospace industry, the space exploration is more and more increasing. The appearance and application of space manipulator is very important for the space on-orbit service, especially about the safety and economy [1-3]. The redundancy of space manipulator improves its flexibility. It can well avoid the singularity and obstacle [4]. At the same time, it brings difficulty to the design and workspace analysis. With the redundant degree increasing, it is more difficult. Now the research of redundant space manipulator is still in an exploration stage. The design and workspace analysis is not clearly solved [5]. The design of a redundant space manipulator with a high working space and independent space is always a hot and difficult problem [6].

In the year of 1975, Roth B presented the concept of workspace firstly [7]. When the each joint of manipulator moves, it refers to a set of all the points that the end effector can achieve. The workspace represents the spatial working extent of redundant space manipulator. It is not only an important kinematic index about its flexibility, but also an important reference for the design and motion planning [8]. There are a lot of methods for solving the problem of workspace, including the graphic method [9], numerical method [10], analytical method [11], simulation method [12] and synthetic method [13]. In a real world application, each of these approaches has advantages and disadvantages. The graphical method is simple and intuitive, but it is only suitable for the 3 degree of freedom and above [14]. The numerical method is more useful, while the calculation is relatively large [15]. The analytical method is relatively complex, and it is not used in the practical application [16]. The simulation and synthetic methods are useful, but the process of debugging is complex [17].

A class of human redundant manipulator is as a research object in this paper. Firstly, the 3D parts of redundant space manipulator are designed and modelled based on SolidWorks 2015. Secondly, the virtual 3D assembly body is established. Thirdly, it is examined by interference and collision. According to the establishment of virtual 3D assembly body, the kinematic equations are established using D-H method. The workspace of human redundant space manipulator is analysed by the Monte Carlo method based on MATLAB R2013a.

\section{3D modelling of redundant space manipulator}

Owing to the flexibility and function, the redundant space manipulator designed in this paper is a kind of human robot arm. It includes a shoulder, upper arm, elbow, wrist, forearm, and end effector. The end effector is like a human hand, which is used to perform the grasping, pulling and lifting. In order to observe the model simply and intuitively, the 3D visualization modelling uses SolidWorks 2015.

\subsection{Design of structural model}

To satisfy the mission requirements of complex space environment, we design a kind of 7 degrees of freedom redundant space manipulator in this paper. There are four common structures, namely S-R-S, U-U-S-A, R-S-S and U-U-S-B. In 1985, J. M. Hollerbach had proved that the structure of S-R-S was the best in the literature [18]. So the structure of S-R-S, also called " $3+1+3$ ", has been 
adopted in this paper. That is the shoulder, elbow and wrist of arm. The 7 degrees of freedom are the rotating joints. Considering the folding of space manipulator, the offset type is adopted in the wrist. The ratio between forearm and upper arm is 1:0.618. The design of structural model is as shown in Figure 1.

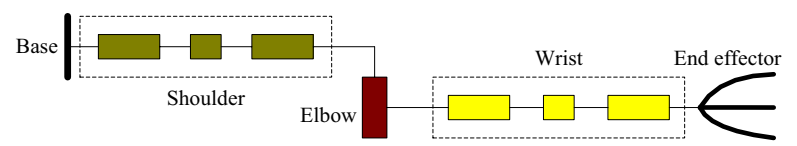

Figure 1. Design of structural model

\subsection{D modelling based on SolidWorks}

In the environment of SolidWorks 2015, the 3D modelling of redundant space manipulator is with the principle of modularization and top-down. First of all, the basic work is the predesign. And then the key work is the design of feature. The core step is to establish the various parts of space manipulator, including the end effector, upper arm, forearm and every joint. Secondly, according to a certain cooperation relationship, the whole machine parts form a virtual assembly. At last, the virtual assembly body is checked by collision and interference. It is used to check the installation position is correct whether or not, to ensure that satisfy the requirements of processing technology. The top-down process of design is as shown in Figure 2.

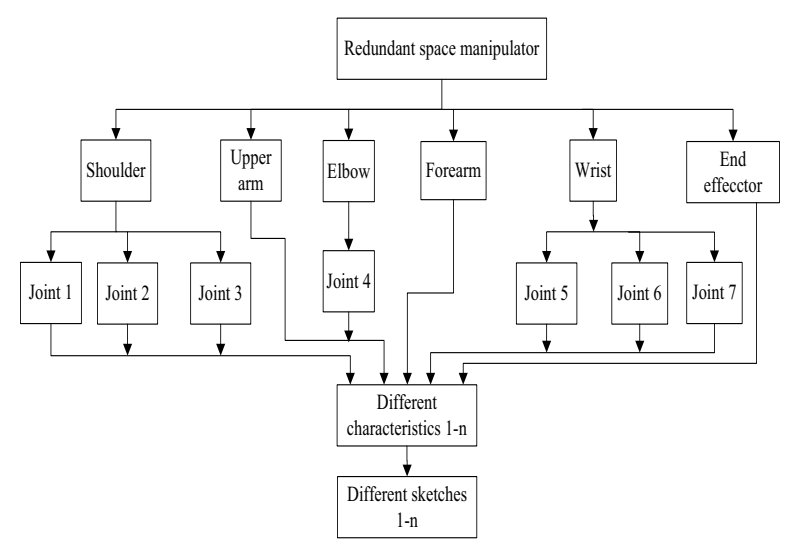

Figure 2. Process of design

The main concrete steps of 3D modelling are as follows.

(1) Modelling of parts

According to the design of structure model, the sketch is drawn based on SolidWorks. The characteristics are established. And the various parts of redundant space manipulator are 3D modelled. They are as shown in Figure 3.

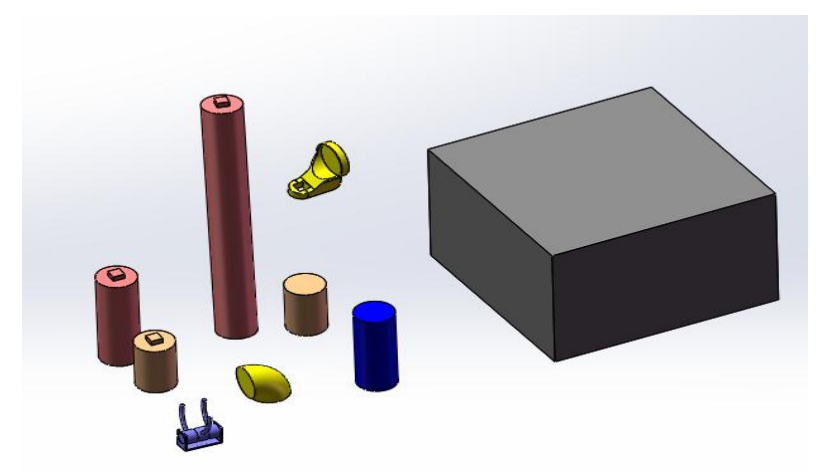

Figure 3. 3D modelling of parts

(2) Build 3D assembly models

The assembly refers to a file. The parts, characteristics and other assembly models are assembled according to the certain relationships. In this paper, there are a total of 23 parts to form a redundant space manipulator. A base is as the first assemble accessory. The assemble relations between them include coincidence and concentric. The whole virtual 3D assembly body is as shown in Figure 4.

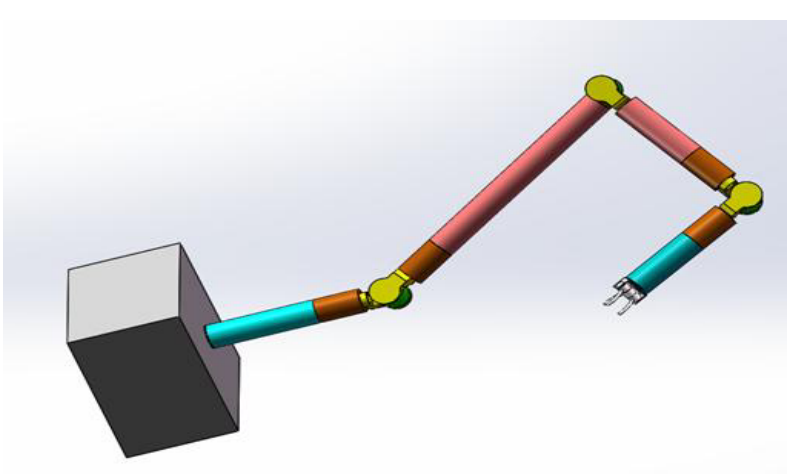

Figure 4. Virtual 3D assembly body

(3) Interference and collision

Due to the complexity of assembly body, it is difficult if only depending on the vision to examine the interference and collision between the parts. Thus, it is examined using the function of interference and collision provided by SolidWorks. This is to check the installation position of the parts in the virtual assembly is correct or nor, to ensure satisfy the conditions of actual processing. In order to observe and analyse the relationship between the various parts, it is generated by the explosion view.

The model of redundant space manipulator has the feature of 3D visualization based on SolidWorks. The 3D model is vividly displayed. We can understand the structure of redundant space manipulator easily and simply. From the whole, it can be had a deeper perception.

\section{Kinematics analysis of redundant space manipulator}

The kinematics analysis of redundant space manipulator is the basis of workspace analysis, motion planning and control. It includes forward and inverse kinematics. The 
forward kinematics is the higher order differential of position, position variable, velocity and acceleration for time or other variables.

\subsection{Establishment of kinematic model}

In order to analyse the kinematic analysis of redundant space manipulator, the D-H method is adopted to describe the joint coordinate system, and then the kinematic model is established. The D-H method is presented by J. Denavit and R. S. Hartenberg in 1995. It has become the standard method in the robot field [19]. Each link of the manipulator can be represented by four kinematic parameters. The rule for describing the relationship of motion with kinematic parameters is named D-H method.

According to the structural model shown in figure 1, the kinematic model of redundant space manipulator is established using D-H method. This is used to describe the relationship between these adjacent bars. Where, $\mathrm{d} 1=0.382 \mathrm{~m}, \mathrm{~d} 3=0.618 \mathrm{~m}, \mathrm{de}=0.08 \mathrm{~m}, \mathrm{~d} 5=\mathrm{d} 7=0.309 \mathrm{~m}$. The joint coordinate system of redundant space manipulator is as shown in Figure 5.

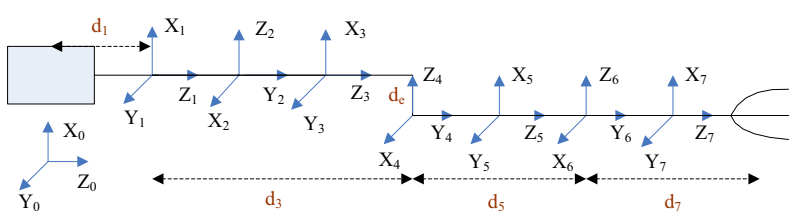

Figure 5. Joint coordinate system of space manipulator

According to the Figure 5, the D-H parameters of redundant space manipulator are as listed in Table 1 . Where, $a_{i-1}, d_{i}$, and $\alpha_{i-1}$ are the three fixed link parameters. $\theta_{i}$ is the joint variable.

Table 1. D-H parameters of redundant space manipulator.

\begin{tabular}{|c|c|c|c|c|}
\hline$i$ & $a_{i-1}(m)$ & $d_{i}(m)$ & $\alpha_{i-1}(\mathrm{rad})$ & $\theta_{i}(o)$ \\
\hline 1 & 0 & $d_{1}$ & 0 & $\theta_{1}$ \\
\hline 2 & 0 & 0 & $\pi / 2$ & $\theta_{2}$ \\
\hline 3 & 0 & $d_{3}$ & $-\pi / 2$ & $\theta_{3}$ \\
\hline 4 & 0 & $-d_{e}$ & $\pi / 2$ & $\theta_{4}$ \\
\hline 5 & 0 & $d_{5}$ & $-\pi / 2$ & $\theta_{5}$ \\
\hline 6 & 0 & 0 & $\pi / 2$ & $\theta_{6}$ \\
\hline 7 & 0 & $d_{7}$ & $-\pi / 2$ & $\theta_{7}$ \\
\hline
\end{tabular}

In Table $1, a_{i-1}$ represents the distance between $Z_{i-1}$ axis and $Z_{i}$ axis along $X_{i-1}$ axis; $d_{i}$ represents the distance between $X_{i-1}$ axis and $X_{i}$ axis along $Z_{i}$ axis; $\alpha_{i-1}$ represents the angle rotated from $Z_{i-1}$ axis to $Z_{i}$ axis along $X_{i-1}$ axis, the forward is anticlockwise; $\theta_{i}$ represents the angle rotated from $X_{i-1}$ axis to $X_{i}$ axis along $Z_{i}$ axis, the forward is anticlockwise.

\subsection{Forward kinematics analysis}

The forward kinematics analysis of redundant space manipulator is the foundation and premise of other research on the technology of space manipulators. The forward kinematics analysis is a process of the transformation from the joint space to Descartes space. Under the case of known linkage parameters and joint variables, the pose of end effector is solved relative to the reference coordinate system.

The general expression of transition matrix between the adjacent coordinate system is as follows [20]:

$$
{ }_{i}^{i-1} T=\left[\begin{array}{cccc}
c_{i} & -s_{i} & 0 & a_{i-1} \\
s_{i} c \alpha_{i-1} & c_{i} c \alpha_{i-1} & -s \alpha_{i-1} & -s \alpha_{i-1} d_{i} \\
s_{i} s \alpha_{i-1} & c_{i} s \alpha_{i-1} & c \alpha_{i-1} & c \alpha_{i-1} d_{i} \\
0 & 0 & 0 & 1
\end{array}\right]
$$

Where, $c_{i}=\cos \left(\theta_{i}\right), s_{i}=\sin \left(\theta_{i}\right), c \alpha_{i-1}=\cos \left(\alpha_{i-1}\right)$, $s \alpha_{i-1}=\sin \left(\alpha_{i-1}\right)$. Combining with the D-H parameters in table 1 , the pose transition matrix can be expressed as follows:

$$
\begin{aligned}
& { }_{1}^{0} T=\left[\begin{array}{cccc}
c_{1} & -s_{1} & 0 & 0 \\
s_{1} & c_{1} & 0 & 0 \\
0 & 0 & 1 & d_{1} \\
0 & 0 & 0 & 1
\end{array}\right] \\
& { }_{2}^{1} T=\left[\begin{array}{cccc}
c_{2} & -s_{2} & 0 & 0 \\
0 & 0 & -1 & 0 \\
s_{2} & c_{2} & 0 & 0 \\
0 & 0 & 0 & 1
\end{array}\right] \\
& { }_{3}^{2} T=\left[\begin{array}{cccc}
c_{3} & -s_{3} & 0 & 0 \\
0 & 0 & 1 & d_{3} \\
-s_{3} & -c_{3} & 0 & 0 \\
0 & 0 & 0 & 1
\end{array}\right] \\
& { }_{4}^{3} T=\left[\begin{array}{cccc}
c_{4} & -s_{4} & 0 & 0 \\
0 & 0 & -1 & d_{e} \\
s_{4} & c_{4} & 0 & 0 \\
0 & 0 & 0 & 1
\end{array}\right] \\
& { }_{5}^{4} T=\left[\begin{array}{cccc}
c_{5} & -s_{5} & 0 & 0 \\
0 & 0 & 1 & d_{5} \\
-s_{5} & -c_{5} & 0 & 0 \\
0 & 0 & 0 & 1
\end{array}\right]
\end{aligned}
$$




$$
\begin{aligned}
& { }_{6}^{5} T=\left[\begin{array}{cccc}
c_{6} & -s_{6} & 0 & 0 \\
0 & 0 & -1 & 0 \\
s_{6} & c_{6} & 0 & 0 \\
0 & 0 & 0 & 1
\end{array}\right] \\
& { }_{7}^{6} T=\left[\begin{array}{cccc}
c_{7} & -s_{7} & 0 & 0 \\
0 & 0 & 1 & d_{7} \\
-s_{7} & -c_{7} & 0 & 0 \\
0 & 0 & 0 & 1
\end{array}\right]
\end{aligned}
$$

Finally, the pose of end effector relative to the base coordinate system is obtained. The transition matrix can be expressed as follows:

$$
{ }_{7}^{0} T={ }_{1}^{0} T_{2}^{1} T_{3}^{2} T_{4}^{3} T_{5}^{4} T_{6}^{5} T_{7}^{6} T=\left[\begin{array}{cccc}
a_{11} & a_{12} & a_{13} & p_{x} \\
a_{21} & a_{22} & a_{23} & p_{y} \\
a_{31} & a_{32} & a_{33} & p_{z} \\
0 & 0 & 0 & 1
\end{array}\right]
$$

The position vector of end effector relative to the base coordinate system can be expressed as follows:

$$
P=\left[\begin{array}{l}
\left(-c_{12345} s_{6}+c_{45} s_{136}+c_{15} s_{246}+c_{12} s_{356}+c_{3} s_{156}-c_{1236} s_{4}+c_{6} s_{134}\right. \\
\left.-c_{146} s_{2}\right) d_{7}+\left(-c_{123} s_{4}+s_{134}-c_{14} s_{2}\right) d_{5}+\left(-c_{12} s_{3}-c_{3} s_{1}\right) d_{e} \\
-c_{1} s_{2} d_{3} \\
\left(-c_{2345} s_{16}-c_{145} s_{36}+c_{5} s_{1246}+c_{2} s_{1356}-c_{135} s_{6}-c_{236} s_{14}-c_{16} s_{34}\right. \\
\left.-c_{46} s_{12}\right) d_{7}+\left(-c_{23} s_{14}-c_{1} s_{34}-c_{4} s_{12}\right) d_{5}+\left(-c_{2} s_{13}+c_{13}\right) d_{e} \\
-s_{12} d_{3} \\
\left(-c_{345} s_{26}-c_{25} s_{46}+s_{2356}-c_{36} s_{24}+c_{246}\right) d_{7}+\left(-c_{3} s_{24}+c_{24}\right) d_{5} \\
-s_{23} d_{e}+c_{2} d_{3}+d_{1}
\end{array}\right]
$$

Where, $\quad c_{i j \cdots k}=c_{i} c_{j} \cdots c_{k}=\cos \left(\theta_{i}\right) \cos \left(\theta_{j}\right) \cdots \cos \left(\theta_{k}\right)$, $s_{i j \cdots k}=s_{i} s_{j} \cdots s_{k}=\sin \left(\theta_{i}\right) \sin \left(\theta_{j}\right) \cdots \sin \left(\theta_{k}\right)$.

\section{Workspace analysis of redundant space manipulator}

The Monte Carlo method is usual for solving the workspace of manipulator. It need rely on the establishment and solution of forward kinematics equation [21]. The workspace of redundant space manipulator can be summarized as a collection of the position vector solutions, which are the end effector relative to the base coordinate system.

\subsection{Overview of Monte Carlo method}

The Monte Carlo method, which originated from the famous needle test in the year of 1777, was also called the statistical simulation method. It is based on probability statistics. By using random number (or pseudo random number), it is a kind of method to solve computational problems. Itls advantages are simple, fast and adaptable. It mainly includes three steps as shown in Figure 6.

Describing the process
of probability $\rightarrow \begin{gathered}\text { Probability } \\ \text { distribution abstracted }\end{gathered} \rightarrow \begin{gathered}\text { Estimated amount } \\ \text { established }\end{gathered}$

Figure 6. Three steps

With the development of science and computer technology, the application of Monte Carlo method is becoming more and more extensive. In mathematical computing, it well solves the problems of multiple integrals, differential integral equations, computation of eigenvalues, and nonlinear equation groups. In practical engineering application, it has a great contribution on statistical physics, aerospace technology, information system, public management, and medical science.

\subsection{Workspace analysis based on Monte Carlo method}

The Monte Carlo method for the degrees of manipulator has no limitation. It is suitable for analysing e workspace of redundant manipulator. The method is simple and easy to implement. It omits the complicated process of solving. The calculation speed is faster. The fundamentals are as follows. First, the range of joint angles is set. Then, each joint angle can randomly obtain different value in the corresponding range. At last, the point set to be reached by the end effector is workspace. The detail solving steps based on MATLAB 2013a are described as follows.

(1) The forward kinematic equation of redundant space manipulator is solved by D-H method. The position vector of end effector relative to the base coordinate system can be expressed as the formula (10).

(2) In the range, the random values $0-1$ can be generated through the function rand $(1, N)$. The value of joint angle can be described as follows:

$$
\theta_{i}=\theta_{\text {imin }}+\left(\theta_{\text {imax }}-\theta_{\text {imin }}\right) * \operatorname{rand}(1, N)
$$

Where, $\mathrm{i}=1-7$, which represents the number of joints. $\theta_{\text {imin }}$, which represents the minimum value of joint angle. $\theta_{\text {imax }}$, which represents the maximum value of joint angle.

(3) The values of joint angle obtained from the step (2) are brought into the formula (10). The point set of end effector can be solved. It can be shown in the graph through MATLAB 2013a. So the workspace of redundant space manipulator is obtained.

All the steps above were performed and simulated on MATLAB 2013a. N=30000, the workspace of redundant space manipulator is as shown in Figure 7.

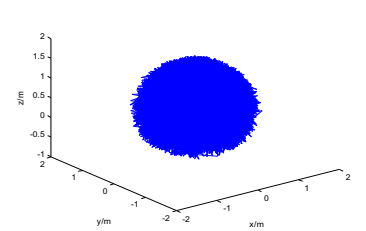

(a) 3D display

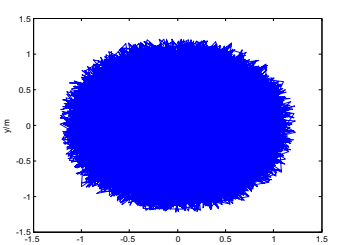

(b) Projection of XOY 


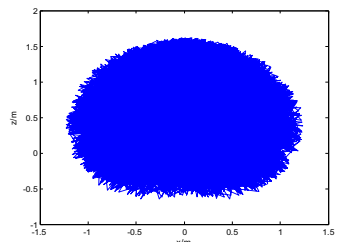

(c) Projection of $\mathrm{XOZ}$

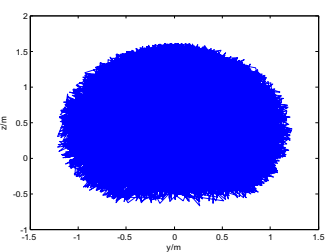

(d) Projection of YOZ
Figure 7. Workspace of redundant space manipulator

From the Figure 7, we can see that the workspace of redundant space manipulator is an oval. It suits the current actual requirement. The whole workspace solved by the Monte Carlo method changes smoothly. What's more, it had not the abrupt phenomenon, and the big hole.

\section{Conclusions}

We designed a kind of 7 degrees of freedom redundant space manipulator in this paper. It is to meet the requirements of tasks in space. Its parts and $3 \mathrm{D}$ modelling are both based on SolidWorks 2015. According to the 3D modelling, the kinematic model is established with the Denavit-Hartenberg (D-H) method. The position vector of end effector relative to the base coordinate system can be solved. The workspace is simulated and analysed using the Monte Carlo method based on MATLAB 2013a. The experimental results showed that the workspace of 7 degree of freedom redundant space manipulator, which changes smoothly. What's more, it had not the abrupt phenomenon, and the independent part is bigger. Thus it verifies the rationality of our designing and parameter selection about the redundant space manipulator. It provides a good reference for the path planning and obstacle avoidance planning.

\section{Acknowledgement}

This work is supported by the National Natural Science Foundation of China (No.61370141, 61300015), the National High Technology Research and Development Program ("863"Program) of China (No. 2015AA2173).

\section{References}

1. E. C. Wu, J. C. Hwang, J. T. Chladek, IEEE Trans. Robot. Autom., 9, 675-684(1993)

2. K. Nagatani, S. Kiribayashi, Y. Okada, K. Otake, K. Yoshida, S. Tadokoro, T. Nishimura, T. Yoshida, E. Koyanagi, M. Fukushima, S. Kawatsuma, J. Field Robot, 30, 44-63(2013)

3. S. Cheng and B. S. Dhillon, J. Quality Maintenance Eng., 17, 203-232(2011)

4. C. J. J. Paredis and P. K. Khosla, J. Robot, 15, 611628(1996)

5. R. G. Roberts, R. J. Jamisola, Jr., A. A. Maciejewski, IEEE Int. Conf. Robot. Autom., 4517-4523(2007)
6. P. S. Naik, A. A. Maciejewski, R. G. Roberts, R. C. Hoover, K. M. Ben-Gharbia, 9th IEEE Int. Conf. Autom. Sci. Eng., 312-317(2013)

7. Y. J. Cui, J. N. Hua, P. Shi, CCTAE 2010, 3(2010)

8. J. Rastegar, B. Fardanesh, J. Mech Mach Theory, 2, 233-239(1990)

9. Y. F. Wang, G. S. Chi, ICRA 2002, 1525-1530(2002)

10. B. Tondu, ICAR 2009, 1-9(2009)

11. F. Zacharias, I. S. Howard, T. Hulin, IROS 2010, 3117-3122(2010)

12. D. Botturi, S. Martelli, P. Fiorini, The $11^{\text {th }}$ $\operatorname{ICARS}(2003)$

13. W. F. Xu, L. T. Li, B. Liang, J. Astronautics, 28, 1389-1394(2007)

14. J. P. Merlet, C. M. Gosselin, N. Mauly, J. Mech. Mach. Theory, 33, 7-20(1998)

15. A. Rezaei, A. Akbarzadeh, P. M. Nia, J. Robotics and Computer-integrated Manufacturing, 29, 158173(2013)

16. D. Pisla, A. Szilaghyi, C Vaida, J. Robotics and Computer-integrated Manufacturing, 29, 463474(2013)

17. J. J. Shao, W. Y. Chen, X. Fu, J. Chin. J. Mech. Engen, 28, 437-450(2015)

18. J. Zhang, Y. Q. Zhao, J. S. Dai, J. Chin. J. Mech. Eng., 27, 703-713(2014)

19. P. K. Artermiadis, P. T. Katsiaris, K. J. Kyriakopoulos, J. Autom. Robots., 29, 293 308(2010)

20. C. Yu, M. Jin, H Liu, ICMA, 92-97(2012)

21. B. F. Li, H. X. Sun, Q. X. Jia, J. Spacecraft Eng., 20, 79-85(2011) 